\title{
A new editor of the JCI
}

\author{
If the rhythm of the drum beat changes, the dance step must adapt. \\ - African proverb
}

This issue of the Journal of Clinical Investigation marks the transition of the position of editor from Gordon Tomaselli to me. It is with great humility that I begin my tenure as the editor of the flagship journal of the American Society for Clinical Investigation (ASCI). On behalf of the $\mathrm{JCl}$ editorial board and editorial staff, I wish Gordon Tomaselli all the best in his new position as the dean of the Albert Einstein College of Medicine.
I have had the opportunity to serve as a deputy editor of the JCI since July 2017, and I am committed to upholding the high standards of the journal that our team has brought to fruition and seek to further our goal of attracting and publishing innovative studies across the spectrum of biomedical research. I bring not only my experiences over the last year with the Johns Hopkins University-based JCI editorial board, but also my service as an associate editor on the editorial board when the JCI was hosted by the University of Pennsylvania, from 2007-2012. I have also served on the editorial boards of Diabetes and Endocrinology, and I served as an associate editor of Gastroenterology, Molecular Endocrinology, and Endocrinology. This up-close vantage point to journal editorship has given me a unique perspective and, I believe, a more nuanced understanding of how the JCI should be positioned in the current publishing landscape and how we can maintain and bolster its reputation for the future.

During the past year, I have been pleased to work with esteemed editorial board colleagues based at Johns Hopkins University, University of Maryland, and the NIH. Under my leadership, the JCI editorial board will remain intact, with the only modifications being that Gordon Tomaselli now serves as a deputy editor, as does Gregg Semenza, Lasker award winner and member of the National Academy of Medicine and National Academy of Sciences. I am delighted that Arturo Casadevall will continue as a deputy editor. Our editorial board meets weekly to discuss manuscripts that are potentially suited for publication in JCI. Every manuscript is assigned a primary handling editor based on subject-matter expertise and a secondary editor from the deputy editor team. These weekly board meetings are the cornerstones of the editorial process and help to ensure consistency among our editorial team.

To date, the current JCI editorial board has handled over 3,200 first-round submissions and accepted 251 papers. During the past year, we have renewed the journal's commitment to publishing high-quality basic and translational science articles, as well as innovative studies in the Clinical Medicine section. We have revamped the Brief Report category, now called Concise Communications, to make the format more flexible for authors and to help attract research findings of immediate interest to the community. We have also made improvements to our publication process across all categories. Authors now have the option to publish in the In-Press Preview format, which allows publication of an unformatted PDF within days of acceptance. Authors may also elect to publish a Graphical Abstract for a quick visual representation of their key findings. Lastly, we recently began allowing the submission of manuscripts posted on preprint servers, and authors can now submit to the JCI directly from the bioRxiv site (https:// www.biorxiv.org/). We believe these flexible options will encourage authors to make their findings more accessible and enhance engagement with the readers.
In an effort to reach out to junior scientists and expand the interest in the ASCI, JCI, and scientific editorial process, we initiated the JCI Scholar's Program. We selected a small number of MD PhD students to shadow associate editors handling manuscripts in their area of study and have given these students the opportunity to participate in the manuscript evaluation process and our editorial board discussion.

During my tenure as JCI editor, I will maintain the progress we have made thus far and place particular emphasis on outreach to authors to ensure that the journal continues to attract cutting-edge research from members of the ASCI and the larger biomedical research community. I believe such efforts are important to sustain traditional areas of strength for the journal, such as oncology, immunology, and metabolism, and to stay competitive in other fields, such as genetics, infectious diseases, pulmonology, cardiovascular medicine, hematology, nephrology, and gastroenterology. I will empower our editorial board members to seek out the best submissions from their colleagues and to ensure that the peer-review process is fair in the scope of revisions requested of authors. I also seek to expand the Viewpoint category that was created by the current editorial board to highlight key findings, controversies, and policy-related issues of interest to the readership. We will continue to highlight areas of interest to our readership through Reviews and Review Series, Commentaries, Author's Take videos, and social media.

The JCI has long served as a leading forum for research of interest to the physician-scientist community, namely basic and clinical studies that improve our understanding of the mechanisms, diagnoses, and treatment of diseases. The JCI faces immense challenges in the current competitive publishing landscape. While recognizing that the popular metrics of journal quality (e.g., impact factors, Eigenfactors) have shortcomings, the JCI cannot ignore the fact that these quality metrics, albeit flawed, influence where authors 
choose to submit their manuscripts. I plan to focus on attracting high-caliber papers in leading biomedical fields to the JCI and on ensuring the selection of the highest quality, most impactful biomedical science through an efficient, fair, and transparent editorial process. Scientific publishing is increasingly dominated by for-profit publishers. Now, more than ever, I believe that it is critical to have active researchers in the field handle your manuscript and to have an editorial board with broad scientific expertise oversee the decision. I believe trust in the content has been the primary factor that has attracted readers to the JCI since its inception.

The JCI has achieved its distinguished status based on its outstanding publications in fundamental science, Clinical Medicine, Reviews and Review Series, and Commentaries pertaining to diseases and health. Our strength is derived collectively from the exemplary service of prior editorial groups, a dedicated editorial staff, ASCI members, and the larger community of biomedical scientists who choose to publish their best work in our journal. As the editor of the JCI, I believe this an excit- ing time to embrace new technologies and methods for biomedical discovery, as well as for the timely transmission of scientific information with the goal of improving human health. I will work tirelessly to ensure that the JCI maintains its status as a premier forum for biomedical research and for providing reliable content using modern platforms with broad appeal to scientists and readers.

\section{Rexford S. Ahima,}

Editor in Chief,

The Journal of Clinical Investigation 